\title{
The higher classification of southern African insects
}

\author{
C.H. Scholtz \\ Scarab Research Group, Department of Zoology E Entomology, University of Pretoria, Private Bag X20, \\ Hatfield, Pretoria, 0028 South Africa.E-mail: chscholtz@zoology.up.ac.za
}

A number of changes have taken place in the higher classification of southern African insects since the last time it was documented in full (Scholtz \& Holm 1985) and there is currently no comprehensive modern classification of higher insect taxa available for the region.

Since 1985 a new order has been discovered in the region (Mantophasmatodea; Klass et al. 2002) and there have been various rearrangements between closely-related groups that have resulted in orders being combined (Blattodea and Isoptera into Blattodea; Inward et al. 2007; Psocoptera and Phthiraptera into Psocodea; Grimaldi \& Engel 2005) while others have been subsumed in larger groups (Hemimerina in Dermaptera; Kocarek et al. 2013). Furthermore, until quite recently Thysanura was the ordinal name used for silverfish or fishmoths but the group is now considered to be paraphyletic with Meinertellidae included; the latter has been moved to the Archaeognatha (Grimaldi \& Engel 2005). Zygentoma was the suborder that included all other thysanurans so it became the default name for the order. Numerous major family-level changes in several large orders have also taken place; the most important being: Coleoptera - Beutel \& Leschen 2005, but modified as in the in press revision of the book; Diptera partially according to Anorim \& Yeates 2006; also Kirk-Spriggs \& Sinclair in press/in prep; Lepidoptera - based largely on Nieukerken et al. 2011; Hymenoptera - bees, Michener 2007, wasps, Pulawski 2012.

These studies bring to 25 the number of insect orders recorded from the region; three others Grylloblattodea, Zoraptera and Rhaphidioptera are absent.

Most of these changes have resulted from molecular phylogenetic analyses which have over the past decade uncovered deeper relationships that are mostly obscured by morphology and behaviour. Nevertheless, there has been strong opposition from some quarters that classification of morphologically and biologically divergent groups need not necessarily reflect hypothesised phylogeny to the letter (Lo et al. 2007). Furthermore, at issue is whether the levels of difference between certain major groups justify according them independence from their close relatives also remain contentious; for example, whether Mantophasmatodea justifies its own order rather than representing an independent African lineage of the Holarctic Grylloblattodea (Terry \& Whiting 2005). However, in spite of resistance, it would seem that most of the changes are becoming entrenched in the literature as the 'accepted' system.

Although modern classifications of most groups are readily accessible on various open-source web sites it remains surprisingly difficult to extract from them the information on which taxa are present in southern Africa. Hence this paper.

Most of these newer published developments were considered when drawing up the following list of higher taxa but I depended heavily on local and foreign specialists on higher classification of different orders to enable me to present a, hopefully, acceptable classification of all southern African higher taxa, from order to family. It should, however, be borne in mind that these classifications in many cases are highly dynamic and more changes will continue to be made over time.

Infraordinal taxon names in bold lettering are not accorded formal status, since the relevant specialists on those groups do not do so; furthermore the taxa lie at different hierarchical levels. Some may be equated to infraorder (i.e. between suborder and superfamily-Hemiptera, Coleoptera), others to a taxon between infraorder and superfamily - Diptera, or to a taxon between superfamily and family - [higher] Hymenoptera.

\section{Order Archaeognatha}

Meinertellidae

\section{Order Zygentoma}

$$
\text { Lepismatidae }
$$

Nicoletiidae

\section{Order Ephemeroptera}

Suborder Pisciforma

Superfamily Siphlonuroidea

Baetidae

Suborder Setisura 
Superfamily Heptagenioidea

Heptageniidae

Oligoneuriidae

Suborder Furcatergalia

Superfamily Ephemerelloidea

Dicercomyzidae

Tricorythidae

Ephemerythidae

Machadorythidae

Teloganodidae

Superfamily Caenoidea

Caenidae

Superfamily Ephemeroidea

Ephemeridae

Euthyplociidae

Polymitarcyidae

Superfamily Leptophlebioidea

Leptophlebiidae

Suborder Carapacea

Prosopistomatidae

\section{Order Odonata}

Suborder Zygoptera

Superfamily Lestoidea

Lestidae

Synlestidae

Superfamily Calopterygoidea

Calopterygidae

Chlorocyphidae

Superfamily Coenagrionoidea

Coenagrionidae

Platyctnemididae

Suborder Anisoptera

Superfamily Aeshnoidea

Aeshnidae

Superfamily Gomphoidea

Gomphidae

Superfamily Libelluloidea

Synthemestidae

Corduliidae

Libellulidae

Macromiidae

Order Plecoptera

Suborder Setipalpia

Perlidae

Suborder Filipalpia

Notonemouridae

\section{Order Embioptera}

Embiidae

Teratembiidae

Archembiidae

Oligotomidae (introduced)
Order Orthoptera

Suborder Ensifera

Superfamily Schizodactyloidea

Schizodactylidae

Superfamily Rhaphidophoroidea

Rhaphidophoridae

Infraorder Gryllidea

Superfamily Grylloidea

Gryllidae

Mogoplistidae

Phalangopsidae

Trigonidiidae

Superfamily Gryllotalpoidea

Gryllotalpidae

Infraorder Tettigoniidea

Superfamily Tettigonioidea

Tettigoniidae

Superfamily Stenopelmatoidea

Gryllacrididae

Stenopelmatidae

Anostostomatidae

Suborder Caelifera

Superfamily Tridactyloidea

Tridactylidae

Superfamily: Tetrigoidea

Tetrigidae

Superfamily Acridoidea

Charilaidae

Acrididae

Pamphagidae

Lathiceridae

Lentulidae

Lithidiidae

Superfamily Eumastacoidea

Eumastacidae

Euschmidtiidae

Thericleidae

Superfamily Pneumoroidea

Pneumoridae

Superfamily Pyrgomorphoidea

Pyrgomorphidae

Order Phasmatodea

Suborder Euphasmatodea

Prisopodidae

Bacillidae

Diapheromeridae

Phasmatidae

Order Dermaptera

Suborder Hemimerina

Hemimeridae

Suborder Forficulina

Pygidicranidae 
Anisolabididae

Labiduridae

Apachyidae

Spongiphoridae (= Labiidae)

Forficulidae

Order Mantophasmatodea

Mantophasmatidae

Austrophasmatidae

Order Blattodea

Epifamily Blattoidae

Superfamily Corydioidea

Corydiidae (= Polyphagidae)

Superfamily Blaberoidea

Ectobiidae (= Blatellidae)

Blaberidae

Superfamily Blattoidea

Blattidae

Epifamily Termitoidae

Hodotermitidae

Kalotermitidae

Rhinotermitidae

Termitidae

Order Mantodea

Empusidae

Hymenopodidae

Mantidae

Sibyliidae

Thespidae

Order Psocodea

Suborder Trogiomorpha

Lepidopsocidae

Trogiidae

Psyllipsocidae

Suborder Troctomorpha

Amphientomidae

Liposcelidae

Group Phthiraptera

Suborder Amblycera

Menoponidae

Boopidae

Laemobothriidae

Ricinidae

Suborder Ischnocera

Philopteridae

Trichodectidae

Suborder Rhynchophthirina

Haematomyzidae

Suborder Anoplura

Echinophthiriidae

Haematopinidae

Neolinognathidae

Enderleinellidae
Hoplopleuridae

Polyplacidae

Hybophthiridae

Ratemiidae

Pedicinidae

Pediculidae

Pthiridae

Linognathidae

Suborder Psocomorpha

Caeciliidae

Elipsocidae

Amphipsocidae

Lachesillidae

Ectopsocidae

Peripsocidae

Pseudocaeciliidae

Mesopsocidae

Hemipsocidae

Myopsocidae

Psocidae

Order Hemiptera

Suborder Auchenorrhyncha Superfamily Cicadoidea

Cicadidae

Superfamily Cercopoidea

Cercopidae

Aphrophoridae

Machaerotidae

Superfamily Cicadelloidea

Membracidae

Cicadellidae

Hylicidae

Superfamily Fulgoroidea

Tettigometridae

Delphacidae

Cixiidae

Fulgoridae

Derbidae

Meenoplidae

Achilidae

Dictyopharidae

Tropiduchidae

Nogodinidae

Hypochthonellidae

Acanaloniidae

Ricaniidae

Lophopidae

Eurybrachiidae

Gengidae

Issidae

Flatidae 
Suborder Sternorrhyncha

Superfamily Psylloidea

Aphalaridae

Calophyidae

Carsidaridae

Liviidae

Phacopteronidae

Psyllidae

Triozidae

Superfamily Aleyrodoidea

Aleyrodidae

Superfamily Aphidoidea

Adelgidae

Phylloxeridae

Aphididae

Superfamily Coccoidea

Aclerdidae

Asterolecaniidae

Cerococcidae

Coccidae

Conchaspididae

Dactylopiidae

Diaspididae

Eriococcidae

Halimococcidae

Kerriidae

Lecanodiaspididae

Margarodidae

Monophlebidae

Ortheziidae

Pseudococcidae

Suborder Heteroptera

Enicocephalomorpha

Enicocephalidae

Dipsocoromorpha

Ceratocombidae

Shizopteridae

\section{Gerromorpha}

Superfamily Mesoveloidea

Mesoveliidae

Superfamily Hebroidea

Hebridae

Paraphrynoveliidae

Superfamily Hydrometroidea

Hydrometridae

Superfamily Gerroidea

Veliidae

Gerridae

\section{Nepomorpha}

Superfamily Nepoidea

Belostomatidae

Nepidae
Superfamily Ochteroidea

Gelastocoridae

Ochteridae

Superfamily Corixoidea

Corixidae

Micronectidae

Superfamily Naucoroidea

Naucoridae

Aphelocheiridae

Superfamily Notonectoidea

Notonectidae

Pleidae

Helotrephidae

Leptopodomorpha

Superfamily Saldoidea

Saldidae

Leptopodidae

\section{Cimicomorpha}

Superfamily Reduvioidea

Reduviidae

Phymatidae

Superfamily Microphysoidea

Microphysidae

Superfamily Miroidea

Thaumastocoridae

Miridae

Tingidae

Superfamily Naboidea

Nabidae

Superfamily Cimicoidea

Lyctocoridae

Anthocoridae

Cimicidae

Polyctenidae

\section{Pentatomorpha}

Superfamily Aradoidea

Aradidae

Superfamily Pentatomoidea

Acanthosomatidae

Cydnidae

Dinidoridae

Pentatomidae

Plataspidae

Scutelleridae

Tessaratomidae

Thaumastellidae

Superfamily Lygaeoidea

Artheneidae

Berytidae

Blissidae

Cymidae

Geocoridae 
Heterogastridae

Lygaeidae

Malcidae

Ninidae

Oxycarenidae

Pachygronthidae

Rhyparochromidae

Piesmatidae

Superfamily Pyrrhocoroidea

Pyrrhocoridae

Superfamily Coreoidea

Alydidae

Coreidae

Rhopalidae

Stenocephalidae

Order Thysanoptera

Suborder Terebrantia

Aeolothripidae

Fauriellidae

Hetrothripidae

Melanthripidae

Merothripidae

Thripidae

Suborder Tubulifera

Phlaeothripidae

Order Megaloptera

Corydalidae

Sialidae

Order Neuroptera

Coniopterygidae

Sisyridae

Osmylidae

Mantispidae

Berothidae

Dilaridae

Psychopsidae

Hemerobiidae

Chrysopidae

Nemopteridae

Ascalaphidae

Myrmeleontidae

Order Strepsiptera

Suborder Mengenillidia

Mengenillidae

Suborder Stylopidia

Corioxenidae

Halictophagidae

Elenchidae

Myrmecolacidae

Stylopidae

Order Coleoptera

Suborder Archostemata
Cupedidae

Micromalthidae

Suborder Myxophaga

Superfamily Sphaeriusoidea

Torridincolidae

Superfamily Sphaeriusoidea

Hydroscaphidae

Sphaeriusidae

Suborder Adephaga

Gyrinidae

Rhysodidae

Carabidae

Haliplidae

Noteridae

Aspidytidae

Dytiscidae

Staphyliniformia

Superfamily Hydrophiloidea

Hydrochidae

Helophoridae

Spercheidae

Epimetopidae

Georissidae

Hydrophilidae

Histeridae

Superfamily Staphylinoidea

Hydraenidae

Ptiliidae

Leiodidae

Silphidae

Staphylinidae

Scarabaeiformia

Superfamily Scarabaeoidea

Bolboceratidae

Passalidae

Trogidae

Glaresidae

Lucanidae

Ochodaeidae

Hybosoridae

Scarabaeidae

Elateriformia

Superfamily Scirtoidea

Eucinetidae

Clambidae

Scirtidae

Superfamily Dascilloidea

Rhipiceridae

Superfamily Buprestoidea

Buprestidae

Superfamily Byrrhoidea

Byrrhidae 


\author{
Elmidae \\ Dryopidae \\ Limnichidae \\ Heteroceridae \\ Psephenidae \\ Ptilodactylidae \\ Superfamily Elateroidea \\ Eucnemidae \\ Throscidae \\ Elateridae \\ Lycidae \\ Lampyridae \\ Cantharidae \\ Derodontiformia \\ Superfamily Derodontoidea \\ Nosodendridae \\ Jacobsoniidae \\ Bostrichiformia \\ Superfamily Bostrichoidea \\ Dermestidae \\ Bostrichidae \\ Ptinidae \\ Cucujiformia \\ Superfamily Lymexyloidea \\ Lymexylidae \\ Superfamily Cleroidea \\ Trogossitidae \\ Thanerocleridae \\ Cleridae \\ Acanthocnemidae \\ Prionoceridae \\ Melyridae \\ Superfamily Cucujoidea \\ Boganiidae \\ Sphindidae \\ Biphyllidae \\ Erotylidae \\ Monotomidae \\ Cryptophagidae \\ Silvanidae \\ Passandridae \\ Phalacridae \\ Laemophloeidae \\ Kateretidae \\ Nitidulidae \\ Bothrideridae \\ Cerylonidae \\ Discolomatidae \\ Endomychidae \\ Coccinellidae \\ Corylophidae \\ Latridiidae
}

Superfamily Tenebrionoidea

Mycetophagidae

Archeocrypticidae

Ciidae

Tetratomidae

Melandryidae

Mordellidae

Ripiphoridae

Zopheridae

Tenebrionidae

Prostomidae

Oedemeridae

Meloidae

Mycteridae

Pyrochroidae

Salpingidae

Anthicidae

Aderidae

Scraptiidae

Superfamily Chrysomeloidea

Disteniidae

Cerambycidae

Megalopodidae

Chrysomelidae

Superfamily Curculionoidea

Anthribidae

Belidae

Attelabidae

Brentidae

Curculionidae

Order Mecoptera

Order Siphonaptera

Bittacidae

Suborder Pulicomorpha

Superfamily Pulicoidea

Hectopsyllidae (= Tungidae; introduced)

Pulicidae

Superfamily Malacopsylloidea

Rhopalopsyllidae

Suborder Hystrichopsyllomorpha

Superfamily Hystrichopsylloidea

Hystrichopsyllidae

Chimaeropsyllidae

Suborder Ceratophyllomorpha

Superfamily Ceratophylloidea

Ceratophyllidae

Ischnopsyllidae

Leptopsyllidae

\section{Order Diptera}

Suborder Tipulomorpha

Limoniidae 
Tipulidae

Suborder Psychodomorpha

Tanyderidae

Ptychopteridae

Blephariceridae

Psychodidae

Suborder Culicomorpha

Thaumaleidae

Simuliidae

Ceratopogonidae

Chironomidae

Dixidae

Corethrellidae

Chaoboridae

Culicidae

Neodiptera

Suborder Bibionomorpha

Bibionidae

Anisopodidae

Mycetophilidae

Sciaridae

Heterotricha-group (Sciaroidea, unassigned to family)

Scatopsidae

Cecidomyiidae

Keroplatidae

Lygistorrhinidae

Suborder Brachycera

Infraorder Stratiomyiomorpha

Xylomyidae

Stratiomyidae

Infraorder Tabanomorpha

Tabanidae

Rhagionidae

Athericidae

Vermileonidae

Infraorder Muscomorpha

Section Nemestrinoidea

Nemestrinidae

Acroceridae

Section Asiloidea

Therevidae

Scenopinidae

Apioceridae

Mydidae

Asilidae

Mythicomyiidae

Bombyliidae

Eremoneura

Section Empidoidea

Empididae

Hybotidae
Brachystomatidae

Atelestidae

Homalocnemiidae

Dolichopodidae

Section Cyclorrhapha

Superfamily Phoroidea

Lonchopteridae

Phoridae

Platypezidae

Superfamily Syrphoidea

Pipunculidae

Syrphidae

Schizophora

Superfamily Sciomyzoidea

Conopidae

Sepsidae

Natalimyzidae

Sciomyzidae

Coelopidae

Superfamily Tephritoidea

Tephritidae

Pyrgotidae

Ctenostylidae

Platystomatidae

Lonchaeidae

Piophilidae

Ulidiidae (Otitidae)

Superfamily Nerioidea

Neriidae

Micropezidae

Superfamily Diopsoidea

Diopsidae

Psilidae

Superfamily Lauxanioidea

Lauxaniidae

Chamaemyiidae

Celyphidae

Superfamily Sphaeroceroidea

Heleomyzidae

Sphaeroceridae

Chyromyidae

Superfamily Opomyzoidea

Opomyzidae

Xenasteiidae

Clusiidae

Marginidae

Odiniidae

Agromyzidae

Aulacigastridae

Neminidae

Asteiidae

Neurochaetidae 
Periscelididae

Superfamily Ephydroidea

Braulidae

Camillidae

Ephydridae

Diastatidae (incl. Campichoetidae)

Curtonotidae

Drosophilidae

Cryptochetidae

Superfamily Carnoidea

Carnidae

Milichiidae

Canacidae

Chloropidae

Calyptratae

Superfamily Muscoidea

Scathophagidae

Anthomyiidae

Fanniidae

Muscidae

Superfamily Hippoboscoidea

Glossinidae

Hippoboscidae

Superfamily Oestroidea

Calliphoridae

Rhiniidae

Sarcophagidae

Tachinidae

Rhinophoridae

Oestridae

\section{Order Trichoptera}

Suborder Annulipalpia

Infraorder Curvipalpia

Superfamily Hydropsychoidea

Ecnomidae

Hydropsychidae

Polycentropodidae

Dipseudopsidae

Psychomyiidae

Xiphocentronidae

Superfamily Philopotamoidea

Philopotamidae

Infraorder Spicipalpia

Superfamily Hydroptiloidea

Glossosomatidae

Hydroptilidae

Suborder Integrepalpia

Infraorder Planitentoria

Superfamily Limnephiloidea

Goeridae

Lepidostomatidae
Pisuliidae

Infraorder Brevitentoria

Superfamily Leptoceroidea

Calamoceratidae

Leptoceridae

Superfamily Sericostomatoidea

Sericostomatidae

Barbarochthonidae

Hydrosalpingidae

Petrothrincidae

\section{Order Lepidoptera}

Suborder Zeugloptera

Superfamily Micropterigoidea Micropterigidae

Suborder Glossata

Superfamily Hepialoidea

Prototheoridae

Hepialidae

Division Monotrysia

Superfamily Nepticuloidea

Nepticulidae

Opostegidae

Superfamily Tischerioidea

Tischeriidae

Superfamily Incurvarioidea

Incurvariidae

Heliozelidae

Division Ditrysia

Superfamily Tinneoidea

Tineidae

Eriocottidae

Psychidae

Schrenchensteiniidae

Gracillariidae

Superfamily Gelechioidea

Oecophoridae

Gelechiidae

Coleophoridae

Cosmopterigidae

Superfamily Copromorphoidea

Copromorphidae

Alucitidae

Carposinidae

Superfamily Yponomeutoidea

Plutellidae

Yponomeutidae

Lyonetiidae

Aegeriidae

Atychiidae

Superfamily Pyraloidea

Hyblaeidae

Thyrididae 
Pyralidae

Anerastiidae

Superfamily Pterophoroidea

Pterophoridae

Superfamily Zygaenoidea

Zygaenidae

Superfamily Cossoidea

Cossidae

Limacodidae

Chrysopolomidae

Superfamily Tortricoidea

Tortricidae

Superfamily Calliduloidea

Pterothysanidae

Callidulidae

Superfamily Uranioidea

Uraniidae

Epiplemidae

Superfamily Geometroidea

Geometridae

Superfamily Hesperioidea

Hesperiidae

Superfamily Papilionoidea

Papilionidae

Pieridae

Lycaenidae

Libytheidae

Nymphalidae

Superfamily Drepanoidea

Drepanidae

Superfamily Bombycoidea

Bombycidae

Eupterotidae

Lasiocampidae

Saturniidae

Superfamily Sphingoidea

Sphingidae

Superfamily Notodontoidea

Notondontidae

Thyretidae

Lymantriidae

Arctiidae

Noctuidae

\section{Order Hymenoptera}

Suborder Symphyta

Superfamily Siricoidea

Siricidae

Superfamily Orussoidea

Orussidae

Superfamily Tenthredinoidea

Tenthredinidae

Argidae
Suborder Apocrita

Section Parasitica

Superfamily Megalyroidea

Megalyridae

Superfamily Stephanoidea

Stephanidae

Superfamily Trigonaloidea

Trigonalidae (Trigonalyidae)

Superfamily Ichneumonoidea

Braconidae

Ichneumonidae

Superfamily Evanioidea

Evaniidae

Gasteruptiidae

Superfamily Proctotrupoidea

Proctotrupidae

Superfamily Diaprioidea

Diapriidae

Superfamily Ceraphronoidea

Ceraphronidae

Megaspilidae

Superfamily Platygastroidea

Platygastridae

Superfamily Cynapoidea

Cynipidae

Figitidae

Ibaliidae

Liopteridae

Superfamily Chalcidoidea

Agaonidae

Aphelinidae

Azotidae

Chalcididae

Elasmidae

Encyrtidae

Eucharitidae

Eulophidae

Eupelmidae

Eurytomidae

Leucospidae

Mymaridae

Ormyridae

Perilampidae

Pteromalidae

Signiphoridae

Tanaostigmatidae

Torymidae

Trichogrammatidae

Section Aculeata

Superfamily Chrysidoidea

Plumariidae

Embolemidae 


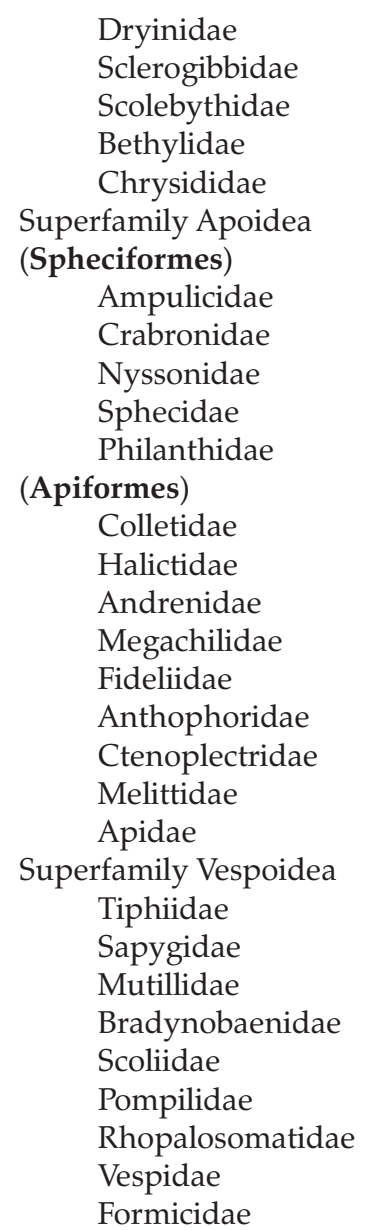

\section{ACKNOWLEGEMENTS}

I am grateful to the following colleagues who provided literature or unpublished information on various taxa. They are listed by order according to the phylogenetic sequence of the groups.

Ephemeroptera - Helen James, Albany Museum, Grahamstown, South Africa.

Odonata - K-D. Dijkstra, Naturalis Biodiversity Center, Leiden, the Netherlands.

Embioptera - Kelly Miller, University of New Mexico, Albuquerque, U.S.A.

Orthoptera - Dan Otte, Academy of Natural Sci-

\section{REFERENCES}

AMORIM, D. de S. \& YEATES, D. 2006. Pesky gnats: ridding dipteran classification of the Nematocera. Studia dipterologica 13(1): 1-7.

BEUTEL, R.G. \& LESCHEN, R.A.B. 2005. Coleoptera, ences, Natural History Museum, Philadelphia, U.S.A.; Corey Bazelet, Department of Entomology and Conservation, University of Stellenbosch, South Africa.

Phasmatodea - Paul Brock, Department of Entomology, The Natural History Museum, London, U.K.

Mantophasmatodea - Mike Picker, University of Cape Town, South Africa.

Blattodea - George Beccaloni, Department of Entomology, The Natural History Museum, London, U.K.

Mantodea - Gavin Svenson, Cleveland Museum of Natural History, Cleveland, U.S.A.

Psocodea (Phthiraptera) - Vince Smith, Department of Entomology, The Natural History Museum, London, U.K.

Hemiptera (Sternorrhyncha) - Ian Millar, National Collection of Insects, Agricultural Research Council, Pretoria, South Africa.

Hemiptera (Heteroptera) - Dawid Jacobs, Department of Zoology and Entomology, University of Pretoria, Pretoria, South Africa.

Megaloptera and Neuroptera - Mervyn Mansell, Department of Zoology and Entomology, University of Pretoria, Pretoria, South Africa.

Coleoptera - Rolf Beutel, University of Jena, Germany; Riaan Stals, National Collection of Insects, Agricultural Research Council, Pretoria, South Africa.

Diptera - Ashley Kirk-Spriggs, National Museum, Bloemfontein, South Africa; Brian Wiegmann, North Carolina State University, Raleigh, U.S.A.

Trichoptera - Helen James and Ferdy de Moor, Albany Museum, Grahamstown, South Africa.

Lepidoptera - Hermann Staude, Lepidopterists Society, South Africa.

Hymenoptera (Parasitica) — Gerhard Prinsloo, National Collection of Insects, Agricultural Research Council, Pretoria, South Africa.

Hymenoptera (Aculeata: bees) - Connal Eardley, National Collection of Insects, Agricultural Research Council, Pretoria, South Africa, and Hymenoptera (Aculeata: wasps) - Sarah Gess, Albany Museum, Grahamstown, South Africa.

Beetles Volume 1: Morphology and systematics In: Kristensen, N.P. \& Beutel, R.G. (Eds) Handbuch der Zoologie Vol. IV (Part 38) Arthropoda: Insecta. Walter de Gruyter, Berlin, Germany. 
GRIMALDI, D. \& ENGEL, M.S. 2005. Evolution of the Insects. Cambridge University Press, Cambridge, U.K.

INWARD, D., BECCALONI, G. \& EGGLETON, P. 2007. Death of an order: a comprehensive molecular phylogenetic study confirms that termites are eusocial cockroaches. Biology Letters 3(3): 331335.

KIRK-SPRIGGS, A.H. \& SINCLAIR B.J. In press. Volume 1. 2016. Manual of Afrotropical Diptera. In prep Volumes 23. Suricata. SANBI, Pretoria, South Africa.

KLASS, K.D., ZOMPRO, O., KRISTENSEN, N.P. \& ADIS, J. 2002. Mantophasmatodea: a new insect order with extant members in the Afrotropics. Science 296(5572): 1456-1459.

KOCAREK, P., JOHN, V. \& HULVA, P. 2013. When the body hides the ancestry: phylogeny of morphologically modified epizoic earwigs based on molecular evidence. PLOS ONE 8(6): e66900 1-9.

LO, N., ENGEL, M.S., CAMERON, S., NALEPA, C.A., TOKUDA, G., GRIMALDI, D., KITADE, O., KRISHNA, K., KLASS, K.D., MAEKAWA, K., MIURA, T. \& THOMPSON, G.J. 2007. Save Isoptera: A comment on Inward et al. Biology Letters 3(5): 562-563.

MICHENER, C.D. 2007. The Bees of the World. 2nd Edition. Johns Hopkins University Press, Baltimore, MD, U.S.A.

NIEUKERKEN, E.J. VAN, KAILA, L., KITCHING, I.J.,
KRISTENSEN, N.P., LEES, D.C., MINET, J., MITTER, C., MUTANEN, M., REGIER, J.C., SIMONSEN, T.J., WAHLBERG, N., YEN, S-H., ZAHIRI, R., ADAMSKI, D., BAIXERAS, J., BARTSCH, D., BENGTSSON, B.A., BROWN, J.W., BUCHELI, S.R., DAVIS, D.R., DE PRINS, J., DE PRINS, W., EPSTEIN, M.E., GENTILI-POOLE, P., GIELIS, C., HÄTTENSCHWILER, P., HAUSMANN, A., HOLLOWAY, J.D., KALLIES, A., KARSHOLT, O., KAWAHARA, A.Y., KOSTER, S. (J.C.), KOZLOV, M.V., LAFONTAINE, J.D., LAMAS, G., LANDRY, J-F., LEE, S., NUSS, M., PARK, K-T., PENZ, C., ROTA, J., SCHINTLMEISTER, A., CHRISTIAN SCHMIDT, B., SOHN, J-C., SOLIS, M.A., TARMANN, G.M., WARREN, A.D., WELLER, S., YAKOVLEV, R.V., ZOLOTUHIN, V.V. \& ZWICK, A. 2011. Order Lepidoptera Linnaeus, 1758. In: Zhang, Z-Q. (Ed.) Animal biodiversity: an outline of higher-level classification and survey of taxonomic richness. Zootaxa 3148: 212-221.

PULAWSKI, W.J. 2012. Catalog of Sphecidae sensu lato: Online at: http://research.calacademy.org/ent/catalog_sphecidae.

TERRY, M.D. \& WHITING, M.F. 2005. Mantophasmatodea and phylogeny of lower neopterous insects. Cladistics 21(3): 240-257. 